\title{
Productos industrializados y su contenido de azúcar, grasas saturadas y sodio para la alimentación complementaria. Lima, Perú
}

\author{
Industrial foods and their sugar, saturated fat and sodium content for \\ complementary feeding. Lima, Peru
}

\author{
Jéssica Verónica León Garay ${ }^{1}$ \\ jessicavleong@gmail.com
}

\author{
María Dextre Jáuregui ${ }^{2}$ ○ \\ mdextrej@unmsm.edu.pe
}

Michelle Fátima Lozada-Urbano ${ }^{3}$ 일

michelle.lozada@uwiener.edu.pe

Artículo recibido: 28/05/2021

Revisado por pares

Artículo aceptado: 24/01/2022

Artículo publicado: 25/02/2022

Autor de correspondencia

Michelle Fátima Lozada-Urbano, michelle.lozada@uwiener.edu.pe

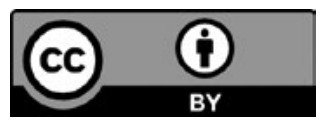

(CLas autoras, 2022. Publicado por la Universidad Norbert Wiener (Lima, Perú)

Citar como: León J, Dextre M, Lozada-Urbano MF. Productos industrializados y su contenido de azúcar, grasas saturadas y sodio para la alimentación complementaria. Lima, Perú. Rev. Inv. UNW. 2022; 11(1): a0002.

doi: https://doi.org/10.37768/unw.rinv.11.01.a0002

\begin{abstract}
Resumen
El objetivo fue describir el contenido nutricional del azúcar, las grasas saturadas y el sodio en productos industrializados para alimentación complementaria (AC), y compararlos con los parámetros técnicos del manual de advertencias publicitarias. El estudio es de tipo descriptivo y diseño transversal con enfoque cuantitativo.

Se trabajó con 46 productos dirigidos a niños entre 6 y 35 meses. La recolección se realizó de marzo a agosto del 2018 en supermercados y farmacias de la ciudad de Lima, Perú. A partir de la etiqueta nutricional del empaque, se obtuvo la cantidad de azúcar, sodio y grasa saturada descrita en su composición, esta información fue llevada a una tabla de Excel y, luego, al programa SPSS 23 para su análisis y comparación. Se trabajaron los siguientes puntos de corte para azúcar, grasas saturadas y sodio: azúcar en alimentos líquidos (AL): $\geq a$ $6 \mathrm{~g} / 100 \mathrm{ml}$, azúcar en alimentos sólidos (AS): $\geq$ a 22,5 g/100 g; grasa saturada $\mathrm{AL}: \geq$ a $3 \mathrm{~g} / 100 \mathrm{ml}$; grasa saturada en AS: $\geq$ a $6 \mathrm{~g} / 100 \mathrm{~g}$; sodio en AL: $\geq$ a 100 $\mathrm{mg} / 100 \mathrm{ml}$, y sodio en AS: $\geq$ a $800 \mathrm{mg} / 100 \mathrm{~g}$.

Concluimos que el 50\% de las marcas cuya consistencia es líquida contiene azúcar que excede los parámetros de las advertencias publicitarias y el 38\% de marcas cuya consistencia es sólida contiene azúcar que excede los parámetros de las advertencias publicitarias. Los productos evaluados con grasa saturada en su composición no presentaron contenido alto; de igual manera, el sodio está presente en todos los productos, pero ninguno excedió los parámetros de las advertencias publicitarias.
\end{abstract}

Palabras clave: azúcar, sodio, grasa saturada, niños, alimentación complementaria

\begin{abstract}
The objective was to describe the nutritional content of sugar, saturated fat and sodium in industrialized products for complementary foods (CF) and to compare them with the technical parameters of the advertising warnings manual. The study was descriptive, cross-sectional with a quantitative approach.

We worked with 46 products aimed at children between six to 35 months old. The collection was from March to August, 2018, in supermarkets and pharmacies in the city of Lima, Peru. From the nutritional label of the packaging, we obtained the amount of sugar, sodium and saturated fat described in its composition. This information was entered in an Excel table and then to the
\end{abstract}

\footnotetext{
${ }^{1}$ Escuela de Nutrición Humana, Universidad Norbert Wiener. Lima, Perú.

${ }^{2}$ Escuela Profesional de Nutrición, Facultad de Medicina, Universidad Nacional Mayor de San Marcos. Lima, Perú.

${ }^{3}$ South American Center for Education and Research in Public Health, Universidad Norbert Wiener. Lima, Perú.
} 
SPSS 23 program for analysis and comparison. The following cut-off points were used for sugar, saturated fat and sodium: sugar in liquid foods (LF): $\geq$ to $6 \mathrm{~g} / 100 \mathrm{ml}$, sugar in solid foods (SF): $\geq$ to $22.5 \mathrm{~g} / 100 \mathrm{~g}$; saturated fat in LF: $\geq$ to $3 \mathrm{~g} / 100 \mathrm{ml}$, saturated fat in SF: $\geq$ to $6 \mathrm{~g} / 100 \mathrm{~g}$; sodium in LF: $\geq$ to $100 \mathrm{mg} / 100$ $\mathrm{ml}$, sodium in SF: $\geq$ to $800 \mathrm{mg} / 100 \mathrm{~g}$.

We concluded that $50 \%$ of the brands of liquid consistency contain sugar which exceeds the parameters of advertising warnings and $38 \%$ of brands of solid consistency contain sugar which exceeds the parameters of advertising warnings. The evaluated products with saturated fat in their composition did not have a high content of it, and sodium was present in all the products, but none exceeded the advertising warning parameters.

Keywords: sugars, sodium, saturated fat, child, complementary feeding

\section{INTRODUCCIÓN}

Los lactantes y niños menores de 5 años que presentan sobrepeso (SP) y obesidad (OB) se incrementaron de 32 millones en 1990 a 41 millones en el 2016. Asimismo, se predice que el número de lactantes y niños pequeños con SP se incrementará a 70 millones para $2025\left(^{1}\right)$. Un estudio realizado en EE. UU. reveló que más del $70 \%$ de los alimentos para niños con demandas específicas de contenido de nutrientes tenían valores altos de azúcar y grasas saturadas $(2,3)$. La presente investigación aborda los componentes de los alimentos, principalmente al azúcar, el sodio y las grasas saturadas, que se encuentran en productos para lactantes y niños pequeños, debido que una inadecuada alimentación y el consumo de estos productos está relacionado con el desarrollo de SP y OB infantil.

A los seis meses de edad, el niño obtiene un desarrollo gastrointestinal, inmune, neurológico y renal $\left({ }^{4}\right)$, y ese es el momento adecuado para iniciar la introducción de alimentos a la dieta del menor. Este proceso, conocido como AC $\left({ }^{5,6}\right)$, abarca el periodo desde los 6 a los 24 meses, según la OMS $\left({ }^{7}\right)$, y tiene como objetivo cubrir los requerimientos de energía y nutrientes, sin dejar de lado la leche materna (LM), además de establecer hábitos de alimentación saludables ${ }^{(8)}$. Los niños, durante su primer año de vida, son considerados como una población en riesgo, porque su alimentación depende significativamente de las elecciones de los padres, quienes en la mayoría de los casos suelen ser influenciados por la industria de alimentos, los medios de comunicación e incluso por algunos profesionales de la salud $\left({ }^{9}\right)$.
Los alimentos complementarios son nutritivos, inocuos y adecuados para los niños, pero en las últimas décadas estas prácticas han cambiado considerablemente, según las diversas culturas $\left({ }^{10}\right)$. Los alimentos fabricados industrialmente se definen como aquellos que transforman productos de origen vegetal, animal, mineral o combinados, a través de diversos procedimientos, para ser destinados a consumo humano ${ }^{(1)}$ ). Las empresas que elaboran alimentos para niños ha crecido significativamente, y su única finalidad es la de "facilitar" la AC para padres de familia que laboran, por ello se llegó a popularizar. Los padres de familia optaron por estos productos y dejaron de usar los alimentos propios de la localidad $\left({ }^{6}\right)$. Los alimentos industrializados pueden tener en su composición cantidades inadecuadas de calorías, así como de sodio, azúcar y grasas saturadas $\left({ }^{12}\right)$, por lo que son inapropiados para los niños $\left({ }^{3}\right)$. Algunos antecedentes de estudios en Sudáfrica mostraron un elevado contenido de azúcar en productos infantiles $\left({ }^{13-19}\right)$, y un $20 \%$ presentaron niveles altos de sodio $\left({ }^{14-16,18}\right)$ y grasas $\left({ }^{19}\right)$.

La etiqueta es el medio por el cual se brinda información nutricional acerca de un producto $\left({ }^{20}\right)$; además, es esencial en su papel como medio de comunicación $\left.{ }^{(21}\right)$. En ese sentido, describiremos el contenido nutricional de las etiquetas de los productos para alimentación complementaria (azúcar, grasas saturadas y sodio), y los compararemos con los parámetros técnicos del manual de advertencias publicitarias.

\section{METODOLOGÍA}

El estudio es de tipo cuantitativo, descriptivo y transversal. 


\section{Población}

La población está conformada por todos los productos destinados a la AC para niños entre 6 y 35 meses de edad. Fueron, en total, 46 productos.

\section{Criterios de selección}

Los criterios de inclusión fueron que la etiqueta del producto exprese claramente que está dirigido a ese grupo de edad, y se incluyeron productos nacionales e importados. Los criterios de exclusión fueron si la etiqueta expresaba que el producto era para niños mayores de 35 meses. Se excluyeron fórmulas infantiles, leche fortificada y electrolitos orales.

Las variables fueron los datos nutricionales de productos destinados para alimentación complementaria para niños de 6 a 35 meses (contenido de azúcar, grasa saturada y sodio), según consistencia de presentación (sólidos y líquidos).

\section{Técnicas e instrumentos de recolección de datos}

Se elaboró una ficha de registro para los alimentos complementarios de consistencia líquida y sólida. Se registraron datos de como nombre del producto, cantidad de nutrientes por cada 100 g, cantidad por porción, y cantidad de grasa saturada, sodio $y$ azúcar.

\section{Procesamiento y análisis de datos}

Se analizaron 46 productos. La información nutricional se obtuvo del empaque, que constituyó la principal fuente de información; esta información fue fotografiada. Los productos fueron comprados en los supermercados de Lima. Este proceso se desarrolló durante el periodo de marzo a agosto del 2018 .

A través del programa SPSS 23 (Statistical Package for the Social Sciences), se analizó y comparó la información con los parámetros del Manual de advertencias publicitarias en los alimentos procesados, establecido por el Decreto Supremo N. ${ }^{\circ}$ 017-2017-SA, que aprueba el Reglamento de la Ley N. ${ }^{\circ} 30021$, Ley de Promoción de la Alimentación Saludable para Niños, Niñas y Adolescentes (tabla 1).

\section{RESULTADOS}

El contenido de azúcar de los productos de consistencia líquida, considerando el plazo de entrada en vigor mayor o igual a $6 \mathrm{~g} / 100 \mathrm{ml}$, mostró que, en el caso de la marca Agú, 5/6 $(83,3 \%)$ de los productos superaron los parámetros técnicos de advertencia publicitaria; en el caso de la marca Gloria, 7/7 (100\%) superaron los parámetros técnicos de advertencia publicitaria. La marca Organic Republic fue la única marca de consistencia líquida que no contenía azúcares, según la información nutricional que refería en el etiquetado. Los productos de la marca Heinz no referían la cantidad de azúcar; sin embargo, en la lista de ingredientes fue mencionado.

Con respecto al contenido de azúcar de los productos de consistencia sólida, considerando el plazo de entrada en vigor mayor o igual a $22,5 \mathrm{~g} / 100 \mathrm{~g}$, en el caso de la marca Cerelac, 2/2 (100\%) de los productos superaron los parámetros

Tabla 1. Parámetros técnicos de advertencias publicitarias y entrada en vigor del manual de advertencias publicitarias

\begin{tabular}{lcc}
\hline \multirow{2}{*}{ Parámetros técnicos } & A los seis 6 meses & Plazo de entrada en vigor \\
\cline { 2 - 3 } & Mayor o igual a $800 \mathrm{mg} / 100 \mathrm{~g}$ & Mayor o igual a $400 \mathrm{mg} / 100 \mathrm{~g}$ \\
\hline Sodio en alimentos sólidos & Mayor o igual a $100 \mathrm{mg} / 100 \mathrm{ml}$ & Mayor o igual a $100 \mathrm{mg} / 100 \mathrm{ml}$ \\
\hline Sodio en bebidas & Mayor o igual a $22,5 \mathrm{~g} / 100 \mathrm{~g}$ & Mayor o igual a $10 \mathrm{~g} / 100 \mathrm{~g}$ \\
\hline Azúcar total en alimentos sólidos & Mayor o igual a $6 \mathrm{~g} / 100 \mathrm{ml}$ & Mayor o igual a $5 \mathrm{~g} / 100 \mathrm{ml}$ \\
\hline Azúcar total en bebidas & Mayor o igual a $6 \mathrm{~g} / 100 \mathrm{~g}$ & Mayor o igual a $4 \mathrm{~g} / 100 \mathrm{~g}$ \\
\hline Grasas saturadas en alimentos sólidos & Mayor o igual a $3 \mathrm{~g} / 100 \mathrm{ml}$ & Mayor o igual a $3 \mathrm{~g} / 100 \mathrm{ml}$ \\
\hline Grasas saturadas en bebidas & Según la normatividad vigente & Según la normatividad vigente \\
\hline Grasas trans &
\end{tabular}


técnicos de advertencia publicitaria. Lo mismo ocurrió con la marca Baby Melts, en la que 1/1 (100\%) de los productos superaron los parámetros técnicos de advertencia publicitaria. Por último, en el caso de la marca Nestum, 3/6 (50\%) de los productos superaron los parámetros técnicos de advertencia publicitaria. Por otro lado, la marca
Mamá Chef fue la única marca de consistencia sólida que no contenía azúcares, según la información nutricional que refería el etiquetado (tabla 2).

Los productos líquidos de la marca Gloria tienen, en promedio, dos veces más azúcar de lo recomendado. Agú líquido y Cerelac sólido tienen un $67 \%$ y un $42 \%$ más de azúcar que lo sugerido.

Tabla 2. Contenido de azúcar (g) de productos destinados a la alimentación complementaria en niños de 6 a 35 meses

\begin{tabular}{|c|c|c|c|c|}
\hline Consistencia & Marca & $\begin{array}{c}\text { Cantidad de productos } \\
\text { por marca }\end{array}$ & Contenido de azúcar (g) & $\begin{array}{c}\text { Exceso en el contenido de } \\
\text { azúcar }\end{array}$ \\
\hline & & & & $>=6 \mathrm{~g} / 100 \mathrm{ml}$ \\
\hline \multirow[t]{5}{*}{ Líquida } & Agú & 6 & $10+/-4,98$ & $66,67 \%$ \\
\hline & Gloria & 7 & $18,33+/-1,74$ & $205,50 \%$ \\
\hline & Heinz* $^{*}$ & 13 & 0 & $0 \%$ \\
\hline & Organic Republic* & 3 & 0 & $0 \%$ \\
\hline & & & & $>=22,5 \mathrm{~g} / 100 \mathrm{~g}$ \\
\hline \multirow[t]{7}{*}{ Sólida } & Ángel & 1 & 13,2 & $0 \%$ \\
\hline & Baby Melts & 1 & 24,5 & $8,89 \%$ \\
\hline & Baby Mum-Mum & 4 & $12,42+/-1,24$ & $0 \%$ \\
\hline & Cerelac** & 2 & 32 & $42,22 \%$ \\
\hline & Mamá Chef* & 1 & 0 & $0 \%$ \\
\hline & Nestum & 6 & $20,83+/-11,21$ & $0 \%$ \\
\hline & Toddler Mum-Mum** & 2 & 20 & $0 \%$ \\
\hline
\end{tabular}

*Ninguno de los productos contiene azúcar.

**Ambos productos contienen la misma cantidad de azúcar.

Tabla 3. Contenido de grasa saturada y sodio de productos destinados a la alimentación complementaria en niños de 6 a 35 meses

\begin{tabular}{lccccc}
\hline Marca & $\begin{array}{c}\text { Cantidad de } \\
\text { productos por } \\
\text { marca }\end{array}$ & $\begin{array}{c}\text { Contenido de } \\
\text { grasas saturadas } \\
\text { (g) }\end{array}$ & $\begin{array}{c}\text { Exceso en el } \\
\text { contenido de } \\
\text { grasas saturadas }\end{array}$ & $\begin{array}{c}\text { Contenido de } \\
\text { sodio (mg) }\end{array}$ & $\begin{array}{c}\text { Exceso en el } \\
\text { contenido de sodio }\end{array}$ \\
\hline Agú** & 6 & 0 & $0 \% \mathrm{~g} / 100 \mathrm{~g}$ & $>100 \mathrm{mg} / 100 \mathrm{ml}$ \\
\hline Gloria & 7 & 0 & $0 \%$ & 0 & $0 \%$ \\
\hline Heinz & 13 & 0 & $0 \%$ & 0 & $0 \%$ \\
\hline Organic Republic & 3 & 0 & $0 \%$ & 0 & $0 \%$ \\
\hline Ángel & 1 & 0 & $>=6 \mathrm{~g} / 100 \mathrm{~g}$ & $0 \% \mathrm{mg} / 100 \mathrm{~g}$ \\
\hline Baby Melts & 1 & $0 \%$ & 6,6 & $0 \%$ \\
\hline Baby Mum-Mum & 4 & 0.5 & $0 \%$ & 60 & $0 \%$ \\
\hline Cerelac ${ }^{*} \dagger$ & 2 & 0 & $0 \%$ & $302+/-53,2$ & $0 \%$ \\
\hline Mamá Chef & 1 & 4.3 & $0 \%$ & 150 & $0 \%$ \\
\hline Nestum & 6 & 0 & $0 \%$ & 0 & $0 \%$ \\
\hline Toddler Mum-Mum* & 2 & 0 & $0 \%$ & $43,8+/-18,2$ & $0 \%$ \\
\hline
\end{tabular}

*Ambos productos contienen la misma cantidad de grasa saturada.

**Los seis productos contienen la misma cantidad de sodio.

† Ambos productos contienen la misma cantidad de sodio. 
Si bien el exceso no es tan grande como en el caso de los productos Gloria, ya estamos hablando de exceso y, por ende, es perjudicial. Finalmente, Baby Melt sólido tiene un 9\% de exceso de azúcar.

Ninguno de los productos de consistencia líquida y sólida evaluados tiene exceso de grasas saturadas o de sodio en su conformación nutricional (tabla 3).

\section{DISCUSIÓN DE RESULTADOS}

\section{Nuestros hallazgos}

Una de las principales contribuciones de la presente investigación fue describir el contenido de azúcar, grasas saturadas y sodio de los productos para alimentación complementaria utilizando los parámetros técnicos del Manual de Advertencias Publicitarias de la Ley de Promoción de la Alimentación Saludable para Niños, Niñas y Adolescentes en el Perú. De las 12 marcas de alimentación infantil investigadas, en 5 de ellas (41\%) se encontraron valores elevados de azúcar. En los productos de consistencia líquida, de cuatro marcas de alimentación infantil, 2 de ellas (50\%) mostraron una elevada cantidad de azúcar. Respecto de los productos de consistencia sólida, en las 8 marcas investigadas, en 3 (38\%) se encontró elevada cantidad de azúcar.

\section{Contenido de azúcar}

Marais, en Sudáfrica (2019), encontró que el 78\% los productos tenían un contenido de azúcar elevado y, de ellos, el $80 \%$ indicaba que contenían azúcar añadido $\left({ }^{13}\right)$. En nuestro estudio, el 50\% de los productos (4 marcas y 12 presentaciones) mostró una elevada cantidad de azúcar, aunque no indicaban si tenían azúcar añadido. Estos resultados se parecen más a los de Koo, en Taiwán (2018), que encontró en el $50 \%$ de productos para niños contenidos elevados de azúcar $\left({ }^{14}\right)$. Igualmente, Calvillo, en México (2013), halló azúcar en exceso en un $52 \%$ de las comidas para el mismo grupo de edad $\left({ }^{18}\right)$. Dos estudios en Australia, en los años 2012 y 2015, de los autores Mehta y Dunford, respectivamente, mostraron que, en el primero, el $75,2 \%$ de alimentos para niños que fueron definidos por los vendedores como no esenciales mostraron alto contenido de azúcar o grasa $\left({ }^{19}\right)$.

\section{Contenido de grasa}

En cuanto al contenido de grasa saturada (g), de los productos seleccionados, ninguna marca mostró exceder los parámetros de advertencias publicitarias. El estudio de Mehta, en Australia (2012), encontró un $75,25 \%$ de los productos con alto contenido de grasa $\left({ }^{19}\right)$. Carstairs, en Reino Unido (2016), revisó recetas infantiles y en ellas encontró que el $26 \%$ eran altas en energía y más del $44 \%$ tenían exceso de proteínas y grasas totales $\left({ }^{22}\right)$. Nuestros hallazgos, probablemente, se deben a que es más frecuente encontrar productos para niños de sabor dulce que salado.

\section{Contenido de sodio}

En cuanto al contenido de sodio de los productos, todos lo tuvieron en su composición, resultado que explica Calvillo en su estudio, pero ninguno tuvo contenido alto en sodio $\left.{ }^{18}\right)$. Sin embargo, en el estudio realizado por Koo, se encontró alto contenido de sodio en un $20 \%$ en productos para niños $\left({ }^{14}\right)$. Igualmente, Maalouf, en Estados Unidos, encontró elevadas concentraciones de sodio en los productos infantiles $\left({ }^{15}\right)$. En nuestro estudio, ningún producto tuvo alto contenido de sodio.

\section{Efecto en la salud del niño}

En este estudio se observó que, entre los productos analizados, el contenido que predomina es el azúcar, y las marcas de consistencia líquida que superan los parámetros considerando el plazo de entrada son Gloria y Agú. Estudios han demostrado que a un $60 \%$ de los lactantes se les brindan alimentos que contienen azúcares añadidos $\left({ }^{23}\right)$. El impacto de un elevado consumo de azúcar en lactantes y niños pequeños está relacionado con el desarrollo de SP y OB 40, por el exceso de calorías que genera el consumo de gran cantidad de azúcar o por consumir calorías extras que se puede almacenar como grasa $\left({ }^{24}\right)$. El azúcar proporciona calorías vacías con pocos nutrientes o fibra dietética, y desplazan a los alimentos ricos en nutrientes, lo que conlleva a niños sobrealimentados y desnutridos a la vez $\left({ }^{25}\right)$, mayor riesgo de diabetes $\left({ }^{26}\right)$ e hígado graso no alcohólico pediátrico $\left({ }^{27}\right)$.

La mayor cantidad de alimentos procesados y ultraprocesados incluyen sacarosa y fructosa. Su 
elevado consumo induce un metabolismo glucídico con alta demanda de insulina, que promueve que su metabolismo esté asociado con problemas metabólicos como estrés oxidativo, aumento de la lipogénesis de novo, dislipidemia, $\mathrm{OB}$ y resistencia a la insulina $\left({ }^{28}\right)$.

El impacto de la ingesta calórica no estaría relacionado con los efectos de la fructosa en sí, sino con el exceso de carga que sobrepasa el umbral de tolerancia fisiológica $\left({ }^{29}\right)$. La fructosa precipita la acumulación de grasa en el hígado, debido tanto al aumento de la lipogénesis como al deterioro de la oxidación de las grasas. Evidencia reciente sugiere que la predisposición al hígado graso está relacionada con el metabolismo de la fructosa por la fructoquinasa $\mathrm{C}$, lo que resulta en el consumo de ATP, el recambio de nucleótidos y la generación de ácido úrico, que median la acumulación de grasa $\left({ }^{30}\right)$.

La hormona leptina circula en proporción a la grasa corporal y funciona como un regulador clave de la ingesta mediante su interacción con los centros hipotalámicos, y así aumenta la saciedad y el gasto energético. A pesar de ello, la OB y la ingesta de fructosa alteran la función de la leptina, lo cual es conocido como resistencia a la leptina $\left({ }^{31}\right)$. Los centros hipotalámicos se vuelven resistentes a su acción y se inhibe la respuesta de saciedad, lo que produce una mayor ingesta de alimentos $\left({ }^{32}\right)$. Investigaciones revelan que un consumo excesivo de fructosa está asociado con niveles altos de leptina en plasma $\left({ }^{33}\right)$ y alteración de la insulina $\left({ }^{34}\right)$.

Además de los efectos de la leptina, la fructosa - a diferencia de la glucosa o almidón- inactiva hormonas intestinales que están relacionadas con la saciedad y es posible que se liberen pocas cantidades de hormonas de la saciedad $\left({ }^{34}\right)$. Los niños que reciben una elevada cantidad de azúcar tienen una ingesta inadecuada de calorías, que les aporta más calorías y ningún nutriente específico, por lo que genera un impacto negativo en la salud infantil $\left.{ }^{(35}\right)$.

La OMS refiere que el consumo de azúcares libres se debería reducir a menos del $10 \%$ de la ingesta calórica total y traería beneficios adicionales si se reduce a menos del 5\% de la ingesta calórica total (36). Esto también se encuentra sustentado por el Reglamento de la Ley N. ${ }^{\circ} 30021$, Ley de Promoción de la Alimentación Saludable para Niños, Niñas y Adolescentes.

Un estudio en Nueva Zelanda, realizado por Katoforis, encontró poca adición de azúcares libres y añadidos en alimentos para niños, salvo en lácteos y bocadillos dulces $\left({ }^{37}\right)$. En el Perú no hay estudios que hayan valorado azúcares añadidos en alimentos infantiles.

Entre las limitaciones tenemos que no existen muchas investigaciones en el Perú para comparar. La revisión de las etiquetas durante la recolección de información mostró a una marca de alimentos que no mencionaba los gramos de azúcar del producto; sin embargo, este si figuraba en la lista de ingredientes. El cero en los resultados de las etiquetas presenta dos posibilidades: que no contiene el elemento o que este no se informa en la etiqueta nutricional.

\section{CONCLUSIONES}

El contenido de azúcar de las marcas de productos infantiles que tuvieron consistencia liquida y sólida mostró que el $50 \%$ y el $38 \%$, respectivamente, exceden las recomendaciones en el manual de advertencias publicitarias. Las grasas saturadas estuvieron dentro del rango, pues ninguno presentó contenido alto según los parámetros de advertencias publicitarias. Igualmente, ninguno presentó un contenido alto de sodio.

Debe existir mayor vigilancia y cumplimiento de los parámetros de advertencia publicitaria en el Perú por parte de las entidades reguladoras; asimismo, las etiquetas deben incluir toda la información que corresponde a los componentes de los productos y ser una referencia para quien los compra.

\section{CONTRIBUCIÓN DE AUTORÍA}

Jéssica Verónica León Garay, María Luisa Dextre y Michelle Fátima Lozada-Urbano son autoras y responsables de la redacción, revisión y aprobación de la versión final del artículo.

POTENCIALES CONFLICTOS DE INTERESES

Ninguno.

\section{FINANCIAMIENTO}

El estudio no tuvo financiamiento. 


\section{REFERENCIAS BIBLIOGRÁFICAS}

1. Organización Mundial de la Salud. Datos y cifras sobre obesidad y sobrepeso. [Internet]. OMS. [citado el 27 oct 2021]. Disponible en: https://www. who.int/es/news-room/fact-sheets/detail/obesityand-overweight

2. Colby S, Johnson L, Scheett A, Hoverson B. Nutrition marketing on food labels. J Nutr Educ Behav. 2010; 42(2): 92-8.

3. World Health Organization. Commercial foods for infants and young children in the WHO European Region. Policy brief on two new reports by the Regional Office for Europe. Copenhagen, Denmark. 2019. WHO; [Cited: Oct 27]. Disponible en: https://www.euro.who.int/_data/assets/pdf_ file/0007/407563/Commercial-Foods-Policy-BriefLowRes.pdf

4. Comité de Lactancia Materna de la Asociación Española de Pediatría. Recomendaciones sobre alimentación complementaria en el lactante amamantado. AEP; 2018.

5. Dalmau J, Moreno JM. Alimentación complementaria: puesta al día. Pediatr Integral. 2017; XXI: 47.e1-47.e4.

6. Owen CG, Martin RM, Whincup PH, Smith GD, Cook DG. Effect of infant feeding on the risk of obesity across the life course: a quantitative review of published evidence. Pediatrics 2005; 115: 136777.

7. Pan American Health Organization. World Health Organization. Guiding Principles for Complementary Feeding of the Breastfed Child. Pan American Health Organization, World Health Organization; Washington DC: 2003.

8. Meneses-Corona A. Alimentación complementaria. Salud y Administración. 2016; 4(9): 1-4.

9. Flores-Huerta S, Martínez-Andrade G, Toussaint G, Adell-Gras A, Copto-García A. Alimentación complementaria en los niños mayores de seis meses de edad. Bases técnicas. Bol Med Hosp Infant Mex. 2006; 63: 129-44.

10. Fondo de las Naciones Unidas para la Infancia. Estado mundial de la infancia 2005. La infancia amenazada. Nueva York: UNICEF; 2005.
11. Muñoz B. Normativa Sanitaria de Alimentos. Área técnica de normas sanitarias Dirección de Higiene AlimentariayZoonosis[Internet].MinisteriodeSalud (MINSA). Dirección General de salud Ambiental. Lima; [citado el 27 oct 2021]. Disponible en: https:// www2.congreso.gob.pe/sicr/cendocbib/con4_uibd. nsf/9F11388EA0C3C78705257C4500638608/\$FILE/ DIGESA-Normativasanitariadealimentos.pdf

12. Centro Nacional de Epidemiología, Prevención y Control de Enfermedades. Boletín epidemiológico del Perú. 2019; 28(40): 1003-4.

13. Marais NC, Christofides NJ, Erzse A, Hofman KJ. Evidence for high sugar content of baby foods in South Africa. S Afr Med J. 2019; 109(5): 328-32.

14. Koo YC, Chang JS, Chen YC. Food claims and nutrition facts of commercial infant foods. PLoS One. 2018; 13(2): e0191982.

15. Maalouf J, Cogswell ME, Bates M, et al. Sodium, sugar, and fat content of complementary infant and toddler foods sold in the United States. 2015. Am J Clin Nutr. 2017; 105(6): 1443-52.

16. Cogswell ME, Gunn JP, Yuan K, Park S, Merritt R. Sodium and sugar in complementary infant and toddler foods sold in the United States. Pediatrics. 2015; 135(3): 416-23.

17. García AL, Raza S, Parrett A, Wright CM. Nutritional content of infant commercial weaning foods in the UK. Arch Dis Child. 2013; 98(10): 793-7.

18. Calvillo A, Cabada X, García K. La alimentación industrializada del lactante y niño pequeño, el nuevo mega negocio. México: El poder del consumidor; 2013

19. Mehta K, Phillips C, Ward P, Coveney J, Handsley E, Carter P. Marketing foods to children through product packaging: prolific, unhealthy and misleading. Public Health Nutr. 2012; 15(9): 176370.

20. Urquiaga I, Lamarca M, Jiménez P, Echeverría G, Leighton F. ¿Podemos confiar en el etiquetado nutricional de los alimentos en Chile? Rev Méd Chile. 2014; 142(6): 775-81.

21.Loria V, Pérez A, Fernández C, Villarino $M$, Rodríguez D, Zurita $\mathrm{L}$. et al. Análisis de las encuestas sobre etiquetado nutricional realizadas en el Hospital La Paz de Madrid durante la 9. ${ }^{a}$ edición del “Día Nacional de la Nutrición”. Nutr. Hosp. 2011; 26(1): 97-106. 
22. Carstairs SA, Craig LC, Marais D, et al. A comparison of preprepared commercial infant feeding meals with home-cooked recipes. Arch Dis Child. 2016; 101: 1037-42.

23. Murray RD. Savoring Sweet: Sugars in Infant and Toddler Feeding. Ann Nutr Metab. 2017; 70(Suppl 3): $38-46$.

24.Zamani R. Azúcar: ¿qué cantidad es saludable para su niño? California: Programa de Salud de Cuidado Infantil de California.

25. Martínez S, Baraldi L, Louzada M, et al. Ultraprocessed foods and added sugars in the US diet: evidence from a nationally representative crosssectional study. BMJ Open 2016; 6: e009892.

26. Fidler N, Braegger C, Bronsky J, Campoy C, Domellöf M, Embleton ND, et. al. Sugar in infants, children and adolescents: a position paper of the European Society for Paediatric Gastroenterology, Hepatology and Nutrition Committee on Nutrition. J Pediatr Gastroenterol Nutr. 2017; 65(6): 681-96.

27. Arenaza L, Medrano M, Oses M, Huybrechts I, Díez I, Henriksson H, et al. Dietary determinants of hepatic fat content and insulin resistance in overweight/ obese children: a cross-sectional analysis of the Prevention of Diabetes in Kids (PREDIKID) study. Br J Nutr. 2019; 121(10): 1158-65.

28. Durazo E. Nivel de azúcares en alimentos y bebidas procesados y su relación con una dieta saludable. PAG Revista Iberoamericana de Producción Académica y Gestión Educativa. 2014; 1(1).

29. Riveros J, Parada A, Pettinelli P. Consumo de fructosa y sus implicaciones para la salud: malabsorción de fructosa e hígado graso no alcohólico. Nutr. Hosp. 2014; 29(3): 491-9.

30.Jensen T, Abdelmalek MF, Sullivan S, Nadeau KJ, Green M, Roncal C, et al. Fructose and sugar: A major mediator of non-alcoholic fatty liver disease. J Hepatol. 2018; 68(5): 1063-75.
31. Shapiro A, Mu W, Roncal C, Cheng K-Y, Johnson R, Scarpace P. Fructose-induced leptin resistance exacerbates weight gain in response to subsequent high-fat feeding. Am J Physiol Integr Comp Physiol. $2008 ; 295,1370-5$

32.Johnson R, Sánchez-Lozada L, Andrews P, Lanaspa M. A historical and scientific perspective of sugar and its relation with obesity and diabetes. Adv Nutr An Int Rev J. 2017; 8: 412-22.

33. Lê KA, Faeh D, Stettler R, et al. A 4-wk high-fructose diet alters lipid metabolism without affecting insulin sensitivity or ectopic lipids in healthy humans. Am J Clin Nutr. 2006; 84(6): 1374-9. doi:10.1093/ ajcn/84.6.1374

34.Tappy L, Lê KA: Health effects of fructose and fructose-containing caloric sweeteners: where do we stand 10 years after the initial whistle blowings? Curr Diab Rep. 2015; 15, 1-12.

35. Organización Mundial de la Salud. Reducción de la ingesta de azúcares libres en adultos para reducir el riesgo de enfermedades no transmisibles. [Internet]. OMS; 2019. [citado el 27 oct 2021]. Disponible en: https://apps.who.int/iris/bitstream/ handle/10665/154587/WHO_NMH_NHD_15.2_ spa.pdf

36. Organización Mundial de la Salud. Nota informativa sobre la ingesta de azúcares recomendada en la directriz de la OMS para adultos y niños. [Internet]. OMS; 2015. [citado el 27 oct 2021]. Disponible en: https://www.who.int/nutrition/publications/ guidelines/sugar_intake_information_note_es.pdf

37. Katiforis I, Fleming EA, Haszard JJ, Hape-Cramond T, Taylor RW, Heath AM. Energy, sugars, iron, and vitamin B12 content of commercial infant food pouches and other commercial infant foods on the New Zealand market. Nutrients. 2021; 13(2): 657. 